\title{
A New Clustering Algorithm for Face Classification
}

\author{
Shaker K. Ali \\ Department of computer science University of Thi-Qar, Thi-Qar, Iraq \\ E-mail: Shalynar@gmail.com \\ Zainab Naser Azeez \\ Department of computer science University of Thi-Qar, Thi-Qar, Iraq \\ E-mail: zainabnaser92@gmail.com \\ Ahmed Abdul-Hussein Ouda \\ Department of computer science University of Thi-Qar, Thi-Qar, Iraq \\ E-mail: ouda1978@gmail.com
}

\begin{abstract}
In This paper, we proposed new clustering algorithm depend on other clustering algorithm ideas. The proposed algorithm idea is based on getting distance matrix, then the exclusion of the matrix points which will be clustered by saving the location (row, column) of these points and determine the minimum distance of these points which will be belongs the group (class) and keep the other points which are not clustering yet. The propose algorithm is applied to image data base of the human face with different environment (direction, angles... etc.). These data are collected from different resource (ORL site and real images collected from random sample of Thi_Qar city population in lraq). Our algorithm has been implemented on three types of distance to calculate the minimum distance between points (Euclidean, Correlation and Minkowski distance). The efficiency ratio of proposed algorithm has varied according to the data base and threshold, the efficiency of our algorithm is exceeded (96\%). Matlab (2014) has been used in this work.
\end{abstract}

Index Terms_-Biometric, Clustering, distance_Euclidean, distance_correlation, distance_Minkowski, face recognition.

\section{INTRODUCTION}

Nowadays, the world is full of data most of which is stored digitally in electronic media, thus providing huge potential for the development of automatic data analysis, classification and retrieval techniques[1]. Cluster analysis is one of the most widely used techniques for exploratory data analysis, with applications ranging from image processing, speech processing, information retrieval and web applications. As a basic tool, clustering has been developed and modified for different application fields, providing many clustering algorithms. In most cases, the number of clusters is an unknown parameter because clustering is unsupervised and the user has very little knowledge about the data. Thus, the evaluation of different clustering algorithms, and the problem of determining the number of clusters, are important research problems in cluster analysis [2]. The purpose of the research is to suggest new clustering algorithm for face classification.

The structured of this paper as follows; the related work has been discussed in section (II).The details of the clustering discussed in section (III). The measurement distance are used in this paper has been discussed in section (IV) and the clustering algorithm has been discussed in section (V) and the reasons for the lack of efficiency has been discussed in section (VI). Detailed the suggested algorithm has been discussed in section(VII) and then discussed the database used in section (VIII). The proposed algorithm has been discussed in section(IX). The results obtained from the clustering algorithm has been discussed in section (X). And the last section discussed the conclusion of this paper.

\section{RELATED WORK}

Alessandra et.al (2005) presented clustering method for automatic biometric template selection. They used Template selection which is reliable method to minimum the number of templates used in a biometric system to account for variations observed in a person's biometric data. The proposed method has great advantage of a substantial save of memory space and computational time for the verification task [3].Rajiv Mukherjee (2007) presented indexing techniques for fingerprint and iris databases. To determine the identity of a person from a large set of identities. This used fingerprint indexing technique based on a combination of minutiae and ridge features. This research proposes three techniques for iris indexing, the first technique is based on iris codes, the second technique utilizes local binary patterns in the iris texture, and the third technique analyzes the iris texture based on a pixel-level difference histogram. K-mean 
algorithm is used in his research [4]. Jassim T.sarsoh et.al (2012) in this research they classifying of human face images based on the graph theory concepts. They propose an effective clustering algorithm. The principle idea of this algorithm depends on the graphic theory by using the definitions and terms of the graph and the tree. Automatic algorithm are used it does not need to give the number of the resulted clusters a priori. In this research project is the algorithm depended on value of threshold. The changing in this value effects results for each cluster, causing a decrease in the efficiency of this algorithm, in addition to that there are some cases that cause low in the efficiency of the algorithm are as follows, face images for more than one person are lied in same cluster. Alternatively, the face images of some person were partitioned in two clusters. This occurred when they use a small value for the constant threshold [5].

\section{CLUSTERING}

Clustering is the task of assigning sets of objects into groups called as clusters so that the objects in one cluster are more similar than the objects in another cluster. Clustering itself is not one specific algorithm, but it is a task which can be performed by various algorithms that differs from each other in their methods of computing/finding the cluster. Clustering is the process of grouping similar image pixels according to some property into one cluster so that the resulting output cluster shows high intra-cluster similarities and low inter-cluster similarities. Clustering process is an unsupervised classification of data points into groups or clusters [6,7].

Clustering is a task which involves a number of stages. The typical clustering process used the following steps [7]:

- Characteristics Representation: In this step the type, dimensions and features of available data are checked. For that it involves processes such as feature selection or feature extraction.

- Similarity Measurement: In this step similarity between data points is measured. Generally various distance measurement methods viz. Euclidian Distance, Mean Square etc. are used to measure similarity between different data points.

- Collecting the data points: In this step the data points are grouped together into clusters, based on similarity measures obtained from the previous step.

- Data abstraction: In this process data is represented with a compact description of individual cluster and furthermore the cluster prototype, i.e. the centroid of the cluster is calculated and used as final representation

- Output Validation: This is an important stage in clustering process. In this step the resulting output clusters are observed to determine whether the resulted output is meaningful or not. It can be done by various methods; either it compares resulted output with a priori structure, or check whether the structure is intrinsically appropriate for data sets or not, or compares two derived outputs with each other and measure their exclusive merits.

\section{MEASUREMENT DistanCE}

There are many methods of measurement as follow:

\section{A. Euclidean distance}

The Euclidean distance function measures the as -thecrow-flies distance. The formula for this distance between a point $X(X 1, X 2$, etc. $)$ and a point $Y(Y 1, Y 2$, etc.) is:

$$
d=\sqrt{\sum_{j=1}^{n}\left(x_{j}-y_{j}\right)^{2}}
$$

Deriving the Euclidean distance between two data points involves computing the square root of the sum of the squares of the differences between corresponding values [8].

\section{B. Minkowski distance}

Minkowski Distance is the generalized metric distance.

$$
d_{x y}=\left(\sum_{k=1}^{d}\left|X_{i k}-X_{j k}\right|^{\frac{1}{p}}\right)^{p}
$$

Note that when $\mathrm{p}=2$, the distance becomes the Euclidean distance. When $\mathrm{p}=1$ it becomes city block distance. Chebyshev distance is a variant of Minkowski distance where $\mathrm{p}=8$ (taking a limit). This distance can be used for both ordinal and quantitative variables $[9,10]$.

\section{Correlation Measure}

The distance correlation measure is based on the Fourier transform, or characteristic function, defined the distance covariance between the random vectors $\mathrm{X}$ and $\mathrm{Y}$ as the nonnegative number $\mathrm{V}(\mathrm{X}, \mathrm{Y})$ defined by [11]:

$$
V^{2}=(X, Y)=\frac{1}{c_{p} c_{q}} \int_{R q} \int_{R p} \frac{|\emptyset x, y(s, t)-\emptyset x(s) \emptyset y(t)|^{2}}{\|s\|^{p+1}\|t\| \|^{q+1}}
$$

Where $|v|$ denotes the modulus of the complex number $\mathrm{v}$ and

$$
c_{p}=\frac{\pi(p+1) / 2}{\Gamma((p+1) / 2)}
$$

The distance correlation between $\mathrm{X}$ and $\mathrm{Y}$ is

$$
R(X, Y)=\frac{V(X, Y)}{\sqrt{V(X, X)} \cdot \sqrt{V(Y, Y)}}
$$

If both $\mathrm{V}(\mathrm{X}, \mathrm{X})$ and $\mathrm{V}(\mathrm{Y}, \mathrm{Y})$ are positive, and defined to be 0 otherwise. 


\section{Clustering Algorithm}

There are two partitions-clustering algorithms. The first algorithm is an automatic partition clustering while the second one is non-automatic partition clustering algorithm [12].

Auto clustering refers to clustering algorithms that have the ability to automatically determine the number of clusters (i.e. without being provided in advance by an input parameter). In most of the clustering algorithms that have been described so far, the number of clusters needs to be specified in advance. The determination of the number of clusters is an important application of clustering techniques as, if the number of clusters can be automatically discovered [12].

Non automatic variation in this type of clustering, the number of clusters must be given a priori by the programmer. The K-means clustering algorithm is a sample of this type. The accuracy of the obtained results depends on the predicted number of clusters chosen by the user when this algorithm is implemented in real dataset [13].

Jassim T.sarsoh et al proposed an automatic partitioning algorithm .This algorithm uses the neighborhood concept and based on the graph theory concepts. The number of the resulted clusters is not given a priori by the user, but it was automatically determined from the given threshold through the implementation them algorithm $[12,14]$.

\section{ALGORITHM 1:}

\section{Pre-Treatment}

1. For each $\mathrm{x}$, we determine adaptive neighborhood $V_{\varepsilon}(\mathrm{x})$

2. For each $\mathrm{x}$, we compute adaptive density $S_{\varepsilon}(\mathrm{x})$

3. Classing the $\mathrm{x}$ according the resulted values of $S_{\varepsilon}$ in descending order in the table (vector)T

4. The point x such that $\boldsymbol{S}_{\varepsilon}(\mathrm{x})=1$ will be put in the class of the isolated points which we call isolated

5. The remained points are put in the table(vector) called $\mathrm{U}$, where $\mathrm{U}=\mathrm{T}$ isolated

\section{GROUPING}

\section{$\alpha$. INITIALIZATION}

$\mathrm{i}=0$

Let $\mathrm{e}$ is the first element in $\mathrm{U}$ $\mathrm{U}=\mathrm{U}-\{\mathrm{e}\}, \mathrm{GO}$ TO $(\beta)$

\section{$\beta$. CREATION OF NEW CLASS} $\mathrm{i}=\mathrm{i}+1$

e create the class $C_{i}$ and attract the element of $V_{\varepsilon}(\mathrm{e})$ in this class, GO TO $(\delta)$

$$
\begin{aligned}
& \gamma \text {. GROUPING } \\
& C_{K}=C_{K} \cup V_{\epsilon}(\mathrm{e})
\end{aligned}
$$

$\delta$. CONTROL

IF $(\mathrm{U}=\varnothing)$ THEN GO TO $(\epsilon)$

ELSE, let $\mathrm{e}$ is the $1^{\text {st }}$ element remaining in $\mathrm{U}$

$$
\mathrm{U}=\mathrm{U}-\{\mathrm{e}\}
$$

- IF there is not exist any $\mathrm{K} \in\{1, \mathrm{i}\}$ such that e $\in C_{K}$, THEN GOTO $(\beta)$

- IF there exit only one $K \in\{1, \mathrm{i}\}$ such that e $\in C_{K}$, THEN GOTO $(\gamma)$

- ELSE e is frontier point and THEN GOTO $(\delta)$

$\epsilon$. PRINT the obtained classes with the family of the isolated point and the frontier points

\section{Clustering Algorithms DefeCts AND THE REASONS FOR THE LACK OF EFFICIENCY:}

There are two main reasons for the low efficiency of classifying algorithms. They are the scattering of data of the one class and partitioning them to more than one class and the overlapping of elements of each class with elements of another class.

Since the first reason is due to the algorithms being depended on the distance threshold to determine elements of the class, which is not accurate since each class has its own threshold, these thresholds vary from one class to another. Therefore, some algorithms come to make the threshold dynamic variable (adaptive) to fix the problem, but it did not improve the efficiency up to be high or absolute one.

The second reason is that since the convergence of a class element to other class elements is due to more than a convergence with itself class elements that is because the similarity with the other elements in some property.

The perfect clustering algorithm is the one that passes two conditions: the first is universal grouping (This algorithm can group all the elements of the same class in one class); the second is preventable (preventing the other elements of other classes to be one element of this specific class).

The solving of this problem is first by assuming a constant number of each class. Then by finding the elements of the first class until the completion of all elements of this class. Finally, by excluding the previous elements from further classifications to minimize the overall number of elements to prevent them from converging with other classes. That is to say, preventing the elements of the class from scattering to another class.

\section{SUGGESTED ALGORITHM}

The idea of classifying algorithms is based on finding the similarity between the set of points which are different from the points themselves. Most of the algorithms are found points of each group at the same 
time; however, the proposed algorithm is differs from the other algorithms by finding the points of each group sequentially after excluding points of the previous groups.

Suppose $(\mathrm{N})$ represents the number of points which will classify; the number of points of each group is (n) and so that the number of groups will be $(\mathrm{N} / \mathrm{n})$. The algorithm can be summarized as the following:

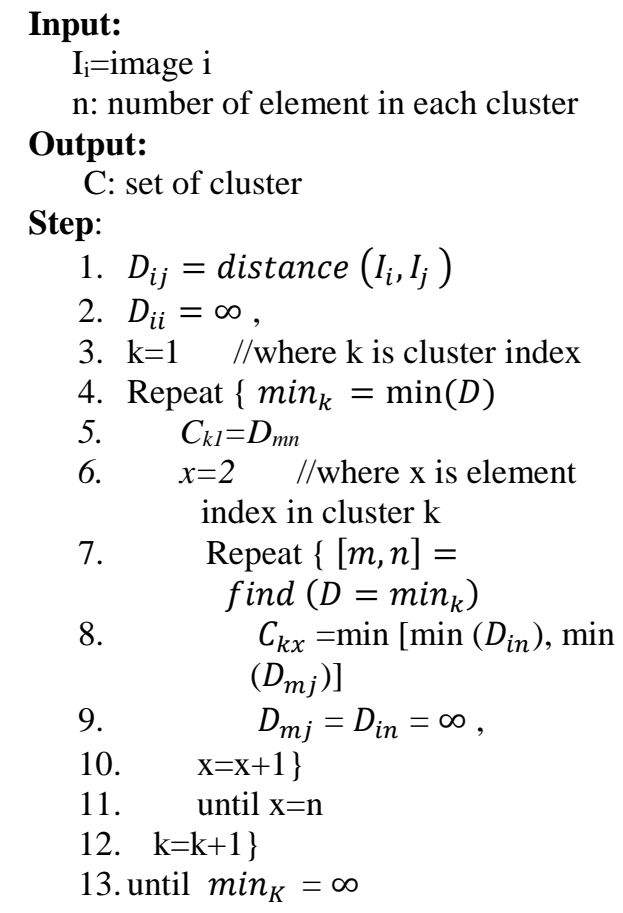

\section{DATABASES}

The proposed approach has been used on different face databases and half face databases. These databases are collected from many different places.

\section{A. ORL Database}

The total images of ORL are (400) where there are 40 persons for each one 10 images were taken. Collecting the image at different times; different lighting; different facial expressions (open and closed eyes, smiling or not, etc.); and different facial details (glasses, mack-up ... ect) in different directions. The size of each image is (92 x112) pixels, with 256 gray levels. This database variable on line [15].

\section{B. Kadhem Mahdi's Database}

This data contains more images than ORL collected from Thi-Qar University. Five face images for each person were taken with different position (vertically and horizontally) and a rotation angle. The database contains face images of both genders with different orientations and simple facial expressions. Some of them are with different facial details [16]. Only 400 images were used from this data.

\section{Research Database}

The database of this research contains only half face of the people whose images were collected from the college of computer science and mathematics. For 144 person with different ages and both genders, the database includes five image for each person with different lighting, different facial expressions (open and closed eye, smiling or not, ... ect) and different facial details (glasses and not, makeup ,... ect), the images were taken from different directions and in different time periods

\section{Proposed ALGORITHM}

The proposed algorithm is consist from 4-steps as following:

\section{A. Data acquisition}

The acquired images used in this paper are collected from 3-data base which mentioned in section (6)-the total image used are (1495) images.

\section{B. PRE-processing}

Pre-processing step include reducing the size of image, remove noise, impurities, and reduce the sharpen images in order to obtain an enhanced image. Which will be suitable for clustering.

\section{Calculate distance matrix}

In this step, the image is converted from a twodimensional matrix into one dimensional matrix, then calculate the distance between each image (test image and target image) to obtain the matrix distance, depend on three types of distance as described in Section (III).

\section{Clustering}

The results from previous steps will produce a symmetrical distance matrix which will be ready to be clustering. Two algorithms are used for clustering in this paper, as described in Section (IV).

\section{EXPERIMENTAL RESUltS}

The results obtained from the clustering algorithm, of different databases and comparing the results from the proposed algorithm with the results from two clustering algorithms are as the following:

\section{1) Using database1 and algorithm1}

By using Euclidean distance and Correlation distance for the database 1 the results are shown in Table 1 . 
Table.1. Efficiency of algorithm1on database1

\begin{tabular}{|c|c|c|c|c|c|}
\hline \multirow{2}{*}{ Experiment No. } & \multirow{2}{*}{$\begin{array}{c}\text { No. Of } \\
\text { image }\end{array}$} & \multicolumn{2}{|c|}{ Correlation dist. } & \multicolumn{2}{c|}{ Euclidean dist. } \\
\cline { 3 - 6 } & 100 & Threshold & Efficiency & Threshold & Efficiency \\
\hline $1^{\text {st }}$ exp. & 100 & 0.72 & $91 \%$ & 0.71 & $92 \%$ \\
\hline $2^{\text {nd }}$ exp. & 100 & 0.75 & $73 \%$ & 0.75 & $82 \%$ \\
\hline 3 th exp. & 100 & 0.70 & $64 \%$ & 0.71 & $85 \%$ \\
\hline $4^{\text {th }} \exp$. & 400 & 0.41 & $70.25 \%$ & 0.65 & $81.75 \%$ \\
\hline $5^{\text {th }} \exp$. & & & & & $81 \%$ \\
\hline
\end{tabular}

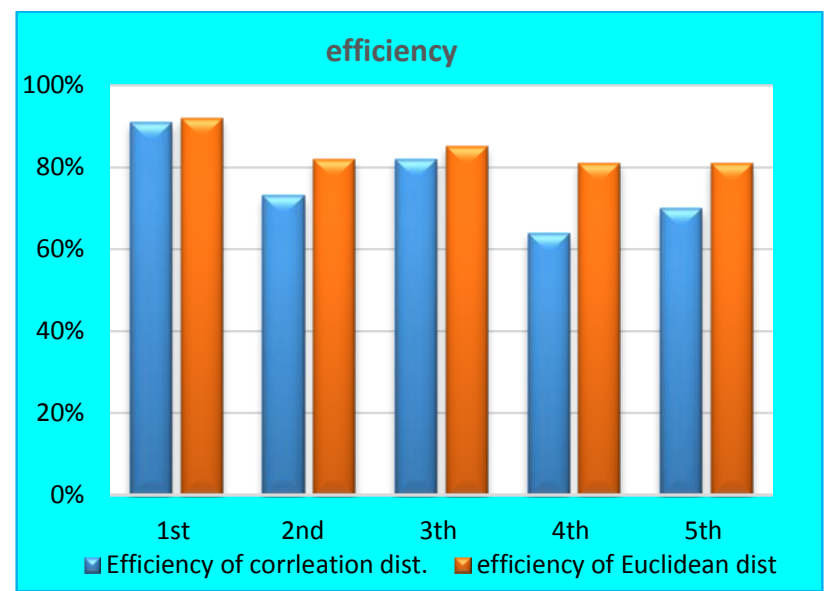

Fig.1. Comparison between efficiency of correlation and Euclidean dist.
The results from the table1 shows the implementation of the algorithm1 by using the database1. By using correlation and Euclidean distance applied to 100 images of 10 persons for each experiment (5 experiments) the efficiency from 5 experiments shows that it's depending on the using correlation distance or Euclidean distance and threshold. As show in table1 and "Fig.1." The best efficiency is by using Euclidean distance.

2) Using Database land Algorithm2 (Suggested Algorithm)

By using Minkowski, Euclidean and correlation distance for the database1 the result are shown in Table 2.

Table.2. Efficiency of algorthim2 on database 1

\begin{tabular}{|c|c|c|c|c|}
\hline Experiment No. & $\begin{array}{c}\text { No. Of } \\
\text { image }\end{array}$ & $\begin{array}{c}\text { Efficiency of } \\
\text { Correlation dist. }\end{array}$ & $\begin{array}{c}\text { Efficiency of } \\
\text { Euclidean dist. }\end{array}$ & $\begin{array}{c}\text { Efficiency of } \\
\text { Minkowski dist. }\end{array}$ \\
\hline $1^{\text {st }}$ exp. & 100 & $86 \%$ & $92 \%$ & $98 \%$ \\
\hline $2^{\text {nd }}$ exp. & 100 & $88 \%$ & $98 \%$ & $100 \%$ \\
\hline 3 th exp. & 100 & $77 \%$ & $100 \%$ & $95 \%$ \\
\hline $4^{\text {th }}$ exp. & 100 & $96 \%$ & $86 \%$ & $89 \%$ \\
\hline $5^{\text {th }}$ exp. & 400 & $79 \%$ & $77 \%$ & $80 \%$ \\
\hline
\end{tabular}

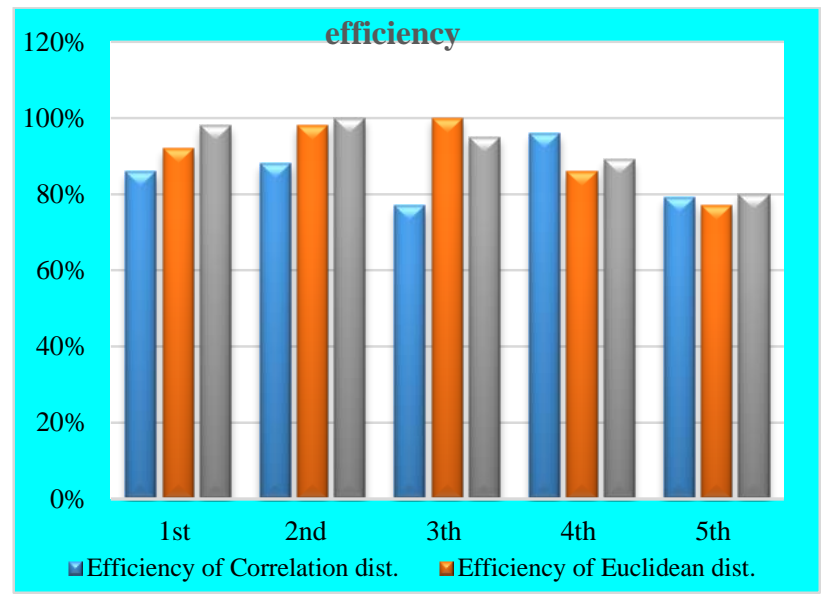

Fig.2. Comparison between efficiency of correlation, Euclidean and minkowski dist.
Table2 shows the results from algorithm2 (the proposed algorithm) with data base1.where 5 experiment are implemented, 100 image (10 image for 10 person) for each experiment. By using three methods (Euclidean, correlation and minkowski) the efficiency from these 5 experiment shows using minkowski is the best results from the others as show in "Fig.2.".

\section{3) Using database 2 and algorithm 1 .}

By using Euclidean and Correlation distance for algorithm1 with database 2 the result are shown in Table 3. 
Table.3. Efficiency of algorthim1on database2

\begin{tabular}{|c|c|c|c|c|c|}
\hline \multirow{2}{*}{ Experiment No. } & \multirow{2}{*}{$\begin{array}{c}\text { No. Of } \\
\text { image }\end{array}$} & \multicolumn{2}{|c|}{ Correlation dist. } & \multicolumn{2}{c|}{ Euclidean dist. } \\
\cline { 3 - 6 } & Threshold & Efficiency & Threshold & Efficiency \\
\hline $1^{\text {st }}$ exp. & 100 & 0.444 & $85 \%$ & 0.646 & $88 \%$ \\
\hline $2^{\text {nd }}$ exp. & 100 & 0.349 & $87 \%$ & 0.569 & $91 \%$ \\
\hline 3 th exp. & 100 & 0.417 & $89 \%$ & 0.589 & $76 \%$ \\
\hline $4^{\text {th }}$ exp. & 100 & 0.517 & $87 \%$ & 0.595 & $72 \%$ \\
\hline $5^{\text {th }}$ exp. & 400 & 0.387 & $85 \%$ & 0.592 & $84 \%$ \\
\hline
\end{tabular}

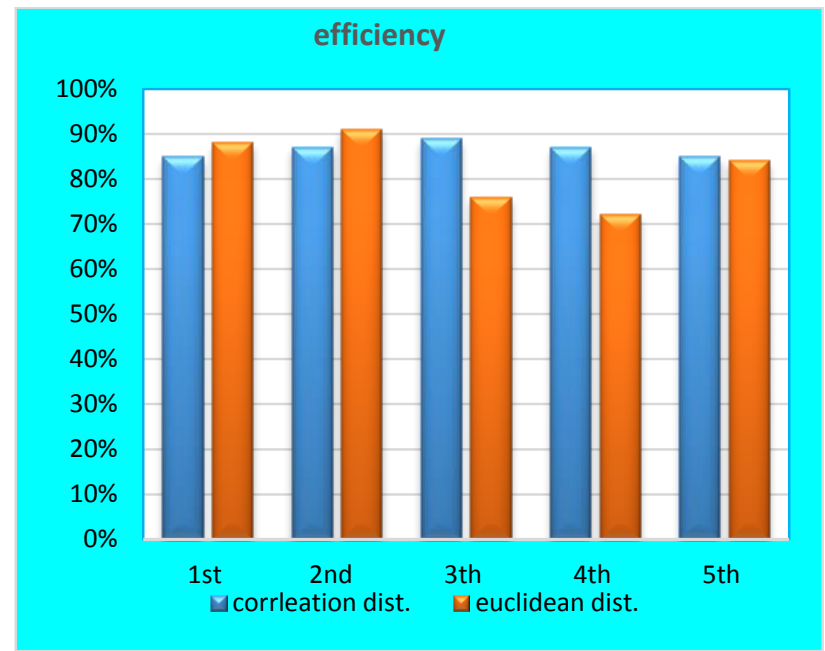

Fig.3. Comparison between efficiency of correlation and Euclidean dist.

Table3 shows the results from algorithm1 and database 2. 5 experiments are implemented for 20 person each person has 5 image for each experiment. By using (correlation, Euclidean distance) the efficiency from these experiments shows that the result from correlations gives best efficiency as show in "Fig.3."

\section{4) Using database 2 and algorithm 2 (proposed} algorithm)

By using Minkowski, Euclidean and Correlation distance for algorithm 2 with the database 2 the result are shown in Table 4

Table.4 shows the results from algorithm2 (the proposed algorithm) with data base 2 .where 5 experiment are implemented, 100 image (5 image for 20 person) for each experiment. By using three methods (Euclidean, correlation and Minkowski) the efficiency from these 5 experiment shows using Minkowski is the best result from the other as show in "Fig.4"

\section{5) Using database3 and algorithm 1}

By using Euclidean and Correlation distance for algorithm1 with database 3 the result are shown in Table 5.

Table.5 shows the results from algorithm1 and database 3. 8 experiments are implemented for 20 person each person has 5 image for each experiment. By using (correlation, Euclidean distance) the efficiency from these experiments shows that the result from correlations gives best efficiency as show in "Fig.5."

Table.4. Efficiency of algorithm2 on database2

\begin{tabular}{|c|c|c|c|c|}
\hline Experiment No. & $\begin{array}{c}\text { No. Of } \\
\text { image }\end{array}$ & $\begin{array}{c}\text { Efficiency of } \\
\text { Euclidean dist. }\end{array}$ & $\begin{array}{c}\text { Efficiency of } \\
\text { Correlation dist. }\end{array}$ & $\begin{array}{c}\text { Efficiency of } \\
\text { Minkowski dist. }\end{array}$ \\
\hline $1^{\text {st }}$ exp. & 100 & $91 \%$ & $77 \%$ & $92 \%$ \\
\hline $2^{\text {nd }}$ exp. & 100 & $90 \%$ & $85 \%$ & $82 \%$ \\
\hline 3 th $\exp$. & 100 & $81 \%$ & $85 \%$ & $86 \%$ \\
\hline $4^{\text {th }} \exp$. & 100 & $78 \%$ & $76 \%$ & $82 \%$ \\
\hline $5^{\text {th }} \exp$. & 400 & $76 \%$ & $73 \%$ & $76 \%$ \\
\hline
\end{tabular}

Table.5. Efficiency of algorithm1 on database3

\begin{tabular}{|c|c|c|c|c|c|}
\hline \multirow{2}{*}{$\begin{array}{c}\text { Experiment } \\
\text { No. }\end{array}$} & \multirow{2}{*}{$\begin{array}{c}\text { No. Of } \\
\text { image }\end{array}$} & \multicolumn{2}{|c|}{ Correlation dist. } & \multicolumn{2}{c|}{ Euclidean dist. } \\
\cline { 3 - 6 } & & Threshold & Efficiency & Threshold & Efficiency \\
\hline $1^{\text {st }}$ exp. & 100 & 0.355 & $84 \%$ & 0.582 & $73 \%$ \\
\hline $2^{\text {nd }}$ exp. & 100 & 0.396 & $81 \%$ & 0.604 & $75 \%$ \\
\hline 3 th exp. & 100 & 0.365 & $78 \%$ & 0.602 & $82 \%$ \\
\hline $4^{\text {th }}$ exp. & 100 & 0.327 & $81 \%$ & 0.576 & $84 \%$ \\
\hline $5^{\text {th }}$ exp. & 100 & 0.304 & $80 \%$ & 0.540 & $85 \%$ \\
\hline $6^{\text {th }}$ exp. & 100 & 0.381 & $84 \%$ & 0.593 & $85 \%$ \\
\hline $7^{\text {th }} \exp$. & 100 & 0.399 & $86 \%$ & 0.618 & $83 \%$ \\
\hline $8^{\text {th }} \exp$. & 700 & 0.30 & $52.52 \%$ & 0.52 & $69.64 \%$ \\
\hline
\end{tabular}




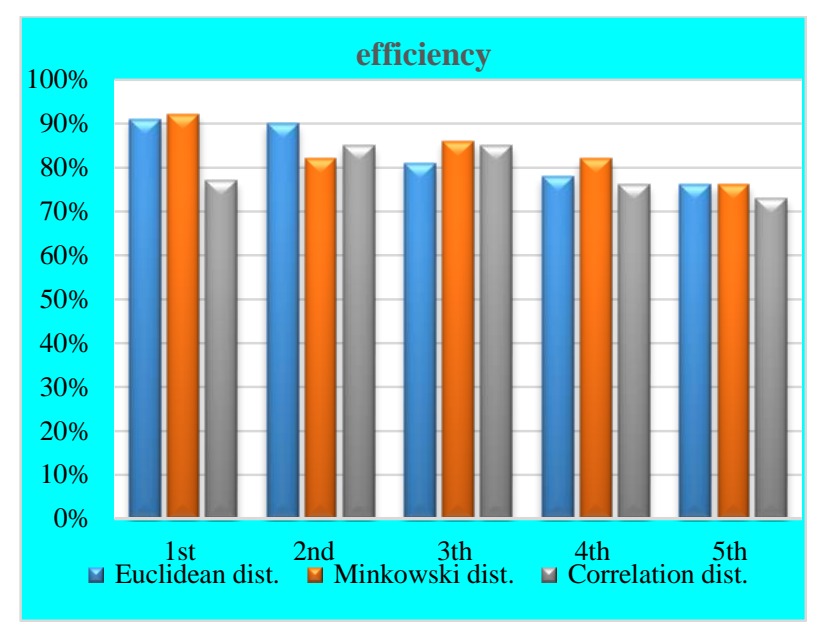

Fig.4. Comparison between efficiency of correlation, Euclidean and minkowski dist.

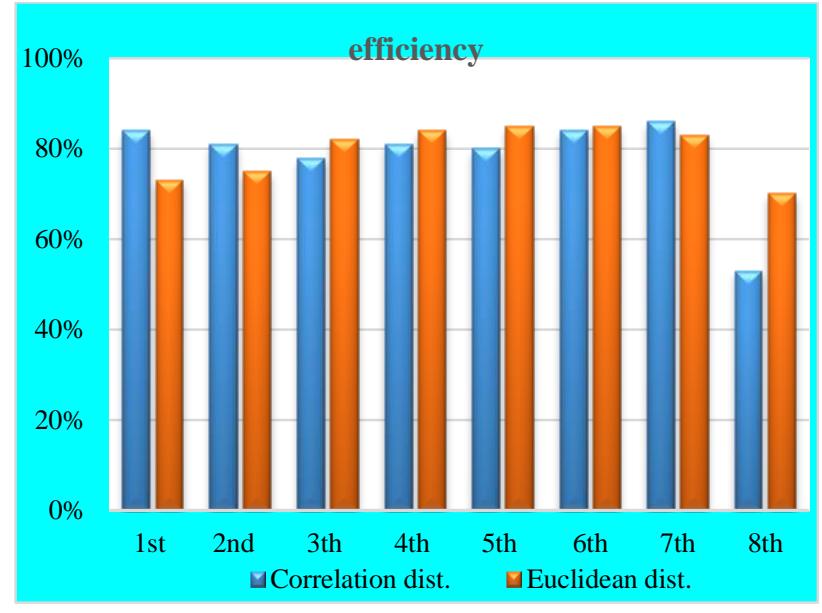

Fig.5. Comparison between efficiency of correlation and Euclidean dist.

Table.6. Efficiency of algorithm3 on database3

\begin{tabular}{|c|c|c|c|c|}
\hline $\begin{array}{c}\text { Experiment } \\
\text { No. }\end{array}$ & $\begin{array}{c}\text { No. Of } \\
\text { image }\end{array}$ & $\begin{array}{c}\text { Efficiency of } \\
\text { Euclidean dist. }\end{array}$ & $\begin{array}{c}\text { Efficiency of } \\
\text { Correlation dist. }\end{array}$ & $\begin{array}{c}\text { Efficiency of } \\
\text { Minkowski dist. }\end{array}$ \\
\hline $1^{\text {st }}$ exp. & 100 & $74 \%$ & $76 \%$ & $78 \%$ \\
\hline $2^{\text {nd }}$ exp. & 100 & $64 \%$ & $66 \%$ & $75 \%$ \\
\hline 3 th $\exp$. & 100 & $78 \%$ & $73 \%$ & $89 \%$ \\
\hline $4^{\text {th }} \mathrm{exp}$. & 100 & $68 \%$ & $70 \%$ & $85 \%$ \\
\hline $5^{\text {th }} \mathrm{exp}$. & 100 & $69 \%$ & $67 \%$ & $85 \%$ \\
\hline $6^{\text {th }} \mathrm{exp}$. & 100 & $73 \%$ & $74 \%$ & $83 \%$ \\
\hline $7^{\text {th }} \mathrm{exp}$. & 100 & $82 \%$ & $81 \%$ & $80 \%$ \\
\hline $8^{\text {th }} \mathrm{exp}$. & 700 & $60.43 \%$ & $63.17 \%$ & $77.27 \%$ \\
\hline
\end{tabular}

\section{6) Using database 3 algorithm2 (proposed algorithm)}

By using Minkowski, Correlation and Euclidean distance for algorithm 2 with database 3 the result are shown in Table 6.

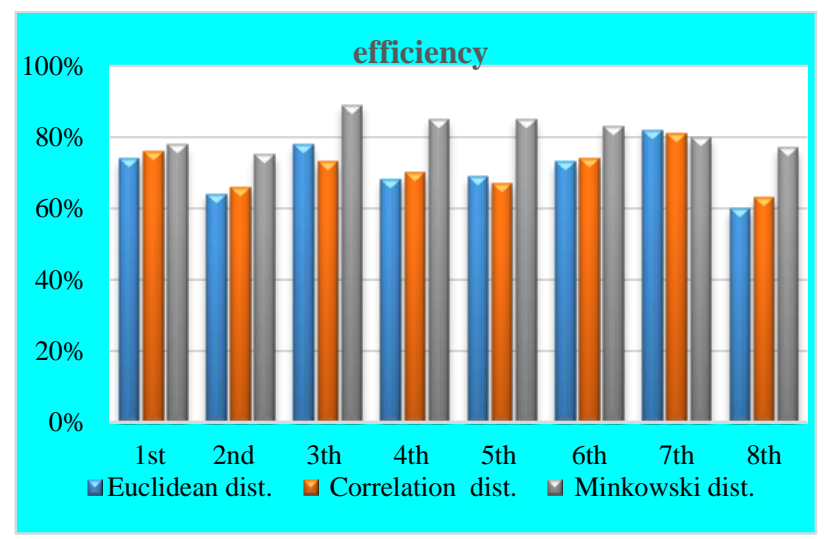

Fig.6. Comparison between efficiency of correlation, Euclidean and minkowski dist

Table.6 shows the results from algorithm 2 (the proposed algorithm) with database 3 .where 8 experiment are implemented, 100 images (5 images for 20 persons) for each experiment. By using three methods (Euclidean, correlation and minkowski) the efficiency from these 8 experiments shows the Minkowski distance is the best result from the others as shown in "Fig.6."

\section{CONCLUSIONS}

The results from algorithm 1 show that when the values of the thresholds are decreasing the dispersion will increase, if the value of threshold be very small value it will may be all images will be a separate cluster. When the value of the threshold is increased the overlapping will increase if the value of threshold be very large it will may be all images are combined in one cluster.

The proposed algorithm will avoid the above problems in algorithm1, where our algorithm will limited specific number of clustering, so it will prevent the dispersion in cluster but the overlapping for closed in distance classes will be occur. It will be cause the effect for efficiency but not very high change or affected the efficiency.

Also the efficiency will be affected from the database images because the huge database will increased the probability of overlapping and big size of one class that will be cluster will increased the likelihood of dispersion.

\section{REFERENCES}

[1] Qinpei Zhao "Cluster Validity in Clustering Methods" $\mathrm{Ph} . \mathrm{D}$. the University of Eastern Finland, Joensuu, on June 25, 2012.

[2] Sindhuja Ranganathan "Improvements to $k$-means clustering" Ph.M. Tampere University of Technology, 14 August 2013.

[3] Alessandra Lumini, Loris Nanni "Aclustering method for automatic biometric template selection " Pattern 
Recognition, Publishers is an imprint of Elsevier 39,495 497, 2006

[4] Rajiv Mukherjee "Indexing Techniques for Fingerprint and Iris Databases" Ph.M.,College of Engineering and Mineral Resources at West Virginia University, 2007.

[5] Jassim T.Sarsoh, Kadhem M.Hashem and Mohammed A.Al-Hadi, " Classifying of Human Face Images Based on the Graph Theory Concepts" Global Journal of Computer Science and Technology Graphics \& Vision, Volume 12 Issue 13 Version 1.0 Year 2012.

[6] Gorti Satyanarayana Murty and V. Vijaya Kumar" Learning Number of Clusters in Unlabeled Dataset using Rotation Estimation" International Journal of Engineering Research and Applications (IJERA) Vol. 2, Issue 2,MarApr 2012, pp.041-045

[7] Ashwini Gulhane, Prashant L. Paikrao," A Review of Image Data Clustering Techniques" International Journal of Soft Computing and Engineering (IJSCE) ISSN: 22312307, Volume-2, Issue-1, March 2012.

[8] T. Soni Madhulatha"An Overview On Clustering Methods" IOSR Journal of Engineering, Apr. 2012, Vol. 2(4) pp: 719-725.

[9] Archana Singh, Avantika Yadav and Ajay Rana"K-means with Three different Distance Metrics" International Journal of Computer Applications (0975 - 8887) Volume 67- No.10, April 2013.

[10] Renato Cordeiro and Boris Mirkin"Minkowski metric, feature weighting and anomalous cluster initializing in KMeans clustering" Elsevier, Pattern Recognition 45, pp.1061-1075.2012.

[11] Elizabeth Martınez-Gomez, Mercedes T. Richards, and Donald St. P. Richards "Distance Correlation Methods for Discovering Associations in Large Astrophysical Databases" The Astrophysical Journal, 781:39 (11pp), 2014.

[12] Jassim T. Sarsoh, Kadhem M. Hashim and Firas S. Miften "Comparisons between Automatic and Non-Automatic Clustering Algorithms" Journal of College of Education for Pure Sciences Vol. 4 No.1, 2011.

[13] M. Ramakrishna Murty and Anima Naik"Automatic Clustering Using Teaching Learning Based Optimization" Scientific Research Publishing, 5.pp. 1202-1211, 2014.

[14] Jassim T.Sarsoh, Kadhem M.Hashem and Mohammed A.Al-Hadi" Classifying of Human Face Images Based on the Graph Theory Concepts" Global Journal of Computer Science and Technology Graphics \& Vision, Vol(12) Issue 13 Version 1.0 Year 2012.

[15] http://www.cl.cam.ac.uk/Research/DTG/attarchive/pub/da ta/att_faces.tar.Z. (Data base for image).

[16] Kadhem M. Hashem "A New Face Recognition Approach using Half Face" Ph.D. University of Basrah, April- 2006.

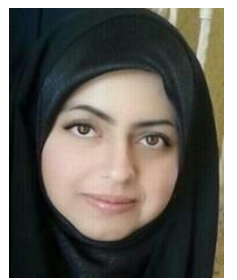

Zainab N. Azeez received her BS degree in computer science from University of ThiQar College of Education for pure science, lraq, in 2013, and currently MS student in computer science from University of ThiQar, her interesting area in Biometric and Clustering algorithms.

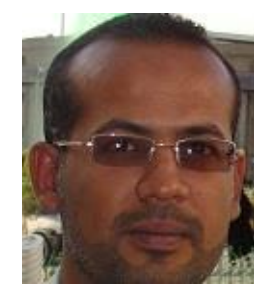

Ahmed Abdul -Hussein Ouda received her BS degree in Nuclear Engineering from Baghdad University, lraq, in 2000, received his MS in Nuclear Engineering from Baghdad University, lraq, in 2004 and currently Ph.D. Student in mechanics engineering University of Basrah. Interesting in php we design, database design, image processing.

How to cite this paper: Shaker K. Ali, Zainab Naser Azeez, Ahmed Abdul-Hussein Ouda, "A New Clustering Algorithm for Face Classification", International Journal of Information Technology and Computer Science (IJITCS), Vol.8, No.6, pp.18, 2016. DOI: $10.5815 /$ ijitcs.2016.06.01

\section{Authors' Profiles}

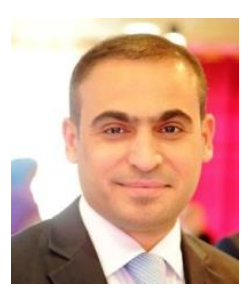

Shaker K Ali received his BS degree in computer engineering from Al- $\mathrm{Al}-$ Mustansiriah University, lraq, in 2000, received his MS degree in computer Science from Al- Technology University, lraq, in 2003, and received his PHD degree from Central South University, Hunan, China. 2011. His interesting area in image processing, Biometric, Artificial intelligent, reviewer for many international journals such as Elsevier. 